\title{
ARTICLE \\ Accelerated development of cocaine-associated dopamine transients and cocaine use vulnerability following traumatic stress
}

\author{
Zachary D. Brodnik ${ }^{1}$, Emily M. Black ${ }^{1}$ and Rodrigo A. España (D)
}

\begin{abstract}
Post-traumatic stress disorder and cocaine use disorder are highly co-morbid psychiatric conditions. The onset of post-traumatic stress disorder generally occurs prior to the development of cocaine use disorder, and thus it appears that the development of post-traumatic stress disorder drives cocaine use vulnerability. We recently characterized a rat model of post-traumatic stress disorder with segregation of rats as susceptible and resilient based on anxiety-like behavior in the elevated plus maze and context avoidance. We paired this model with in vivo fast scan cyclic voltammetry in freely moving rats to test for differences in dopamine signaling in the nucleus accumbens core at baseline, in response to a single dose of cocaine, and in response to cocaine-paired cues. Further, we examined differences in the acquisition of cocaine self-administration across groups. Results indicate that susceptibility to traumatic stress is associated with alterations in phasic dopamine signaling architecture that increase the rate at which dopamine signals entrain to cocaine-associated cues and increase the magnitude of persistent cue-evoked dopamine signals following training. These changes in phasic dopamine signaling correspond with increases in the rate at which susceptible rats develop excessive cocaine-taking behavior. Together, our studies demonstrate that susceptibility to traumatic stress is associated with a cocaine use-vulnerable phenotype and suggests that differences in phasic dopamine signaling architecture may contribute to the process by which this vulnerability occurs.
\end{abstract}

Neuropsychopharmacology (2020) 45:472-481; https://doi.org/10.1038/s41386-019-0526-1

\section{INTRODUCTION}

Post-traumatic stress disorder (PTSD) and cocaine use disorder are highly co-morbid psychiatric conditions [1-3]. The onset of PTSD generally occurs prior to the development of cocaine use disorder, and this has led to the hypothesis that the development of PTSD drives cocaine use vulnerability [4]. The biological substrates of cocaine use vulnerability following PTSD onset, however, remain unclear.

The formation and maintenance of psychological and neurobiological associations between drugs and drug-predictive cues is a critical component in the development and progression of cocaine use disorder $[5,6]$. Such Pavlovian associations are known to trigger cocaine craving [5, 7], and it has been found that PTSD symptom severity predicts the intensity of cue-elicited cocaine craving [8]. Furthermore, cocaine use disorder patients with a history of trauma display elevations in mesolimbic responses to cocaine-predictive cues [9]. This evidence suggests that differences in the development or persistence of Pavlovian associations between cues and cocaine may contribute to cocaine use vulnerability following PTSD onset.

Pavlovian associations develop across repeated cue pairings such that cocaine-associated cues become sufficient to elicit cocaine seeking $[10,11]$. A distributed circuit orchestrates cueelicited cocaine-seeking $[6,12]$, and the nucleus accumbens core (NAc) is an essential node within this circuit [13-15]. In particular, phasic increases in NAc dopamine (DA) appear to play a critical role in cue-elicited drug seeking. For example, it has been shown that phasic DA signals in the NAc correlate with the expression of cocaine-seeking behaviors $[11,16,17]$, and mimicking these phasic DA events by electrical stimulation of DA neurons initiates cocaine seeking [16]. Together these observations raise the possibility that changes in the development and/or persistence of cue-evoked DA signals in response to cocaine-cue associations may drive vulnerability to developing cocaine use disorder in PTSD patients.

We recently described and validated a version of the predator odor stress model of PTSD with segregation of rats as susceptible or resilient based on anxiety-like behavior in the elevated plus maze and context avoidance [18]. Using this paradigm, we found that susceptible rats express a PTSD-like phenotype as well as multiple changes in baseline DA signaling, cocaine pharmacology, and the behavioral economics of cocaine self-administration [18]. Other studies using stress and segregation approaches subsequently demonstrated that susceptible rats display increased cocaine self-administration, attenuated extinction of self-administration, and increases in cue-primed reinstatement of cocaine seeking $[19,20]$. Together these observations point to the cooccurrence of a PTSD-like and cocaine use vulnerability phenotype in susceptible rats, thus affording the opportunity to examine the neurobiology underlying this comorbidity. In the current studies, we tested the hypothesis that susceptibility to traumatic stress is associated with aberrations in phasic DA signaling that contribute

${ }^{1}$ Department of Neurobiology and Anatomy, Drexel University College of Medicine, 2900W Queen Lane, Philadelphia, PA 19129, USA

Correspondence: Rodrigo A. España (rae39@drexel.edu)

Received: 27 February 2019 Revised: 6 September 2019 Accepted: 11 September 2019

Published online: 20 September 2019 
to cocaine use vulnerability. We used fast scan cyclic voltammetry (FSCV) in freely moving rats to examine changes in both spontaneous and cue-elicited phasic DA signals in the NAC following traumatic stress. We then characterized changes in the propensity to develop cocaine-taking behaviors to determine if observed changes in phasic DA signaling correspond with increases in cocaine use vulnerability.

\section{METHODS}

Animals

Male Sprague-Dawley rats (300-350 g, Harlan, Frederick, MD) were given ad libitum access to food and water and kept on a reverse 12:12 h light:dark cycle (lights on at 15:00 or 17:00 h). All protocols and animal care procedures were maintained in accordance with the National Research Council's Guide for the Care and Use of Laboratory Animals: Eighth Edition (The National Academies Press, Washington, DC, 2011) and approved by the Institutional Animal Care and Use Committee at Drexel University College of Medicine.

\section{Chemicals}

Cocaine hydrochloride was obtained from the National Institute on Drug Abuse. 2,4,5-trimethyl-3-thiazoline (TMT), and butyric acid were obtained from Sigma-Aldrich (St. Louis, MO).

\section{Intravenous catheterization surgery}

All rats were implanted with jugular catheters for intravenous (IV) delivery of cocaine. Catheterization surgeries were performed as previously described [18, 21]. For FSCV experiments, rats were anesthetized using isoflurane and for self-administration experiments rats were anesthetized with ketamine $(100 \mathrm{mg} / \mathrm{kg})$ and xylazine $(10 \mathrm{mg} / \mathrm{kg})$. IV silastic catheters were implanted into the right jugular vein. The catheter was connected to a cannula which exited through the skin on the dorsal surface in the region of the scapulae. Rats received post-surgical antibiotic (Neo-Predef, Pharmacia \& Upjohn Company, and $5 \mathrm{mg} / \mathrm{kg}$ Baytril, Bayer HealthCare LLC) and analgesic ( $5 \mathrm{mg} / \mathrm{kg}$; Ketoprofen, Patterson Veterinary) and were allowed 1-3 weeks to recover before experimental procedures began. Catheters were manually flushed with saline every 2-3 days during recovery to maintain catheter patency.

\section{FSCV surgery}

For the subset of rats tested with FSCV, electrodes were implanted immediately following catheterization surgeries. Chronically implanted carbon fiber electrodes were implanted in the NAc core $(+1.0 \mathrm{~A} / \mathrm{P}, \pm 2.3 \mathrm{M} / \mathrm{L},-7.0$ to $-7.5 \mathrm{D} / \mathrm{V})$, and a reference electrode was implanted in the posterior cortex $(-2.5 \mathrm{~A} / \mathrm{P},-2.5 \mathrm{M} / \mathrm{L}$, $-2.0 \mathrm{D} / \mathrm{N})$. Electrodes were secured into place using dental acrylic cement and five screws driven into the skull. Rats received postsurgical antibiotic (Neo-Predef, Pharmacia \& Upjohn Company, and $5 \mathrm{mg} / \mathrm{kg}$ Baytril, Bayer HealthCare LLC) and analgesic ( $5 \mathrm{mg} / \mathrm{kg}$; Ketoprofen, Patterson Veterinary) and were allowed to recover for 21-28 days before FSCV experiments began [22].

\section{Stress and segregation testing}

For all rats, odor exposures, as well as context avoidance and elevated plus maze testing, were carried out as previously described [18]. Treatment of control and stressed rats differed only in the odor of exposure, otherwise all rats underwent the same series of procedures. Control rats were exposed to butyric acid and stressed rats were exposed to TMT. A subset of TMTexposed rats display a PTSD-like phenotype comprised of significant context avoidance, as well as increases in anxiety-like behavior, acoustic startle response, and late waking phase corticosterone levels [18].

Odor exposure was performed in a three-chamber place conditioning box (Med Associates Inc., Fairfax, VT). On the first day of testing, rats were allowed to freely explore all chambers of the apparatus in a recorded, 5-min preference test. On the following day, rats were randomly confined to one of the two context chambers and $10 \mu \mathrm{l}$ of either TMT or butyric acid was pipetted onto tissue paper placed below the chamber floor. Odor exposures were performed at the beginning of the light phase (ZT 0:00) and lasted for $15 \mathrm{~min}$.

Elevated plus maze and context avoidance tests were performed 7 days after odor exposure [18, 20, 23, 24]. Rats were first recorded for 5-min on the elevated plus maze [25]. Time spent in each arm of the elevated plus maze was quantified using Ethovision XT software (Noldus, USA, VA, Leeburg). One hour after elevated plus maze testing, rats were re-introduced to the place conditioning box where they were allowed to freely explore all three chambers in a recorded, 5-min context avoidance test. To quantify context avoidance behavior, we calculated the change in time spent in the odor-paired chamber ( $\Delta$ Paired), which was defined as the difference in time spent in the odor-paired chamber during the avoidance and preference tests. Nonspecific avoidance ( $\Delta$ Unpaired) was similarly calculated for time spent in the unpaired chamber. All animals included in these studies underwent this series of procedures and tests, and we refer to this process as "Stress and Segregation" testing (Fig. 1a).

To determine if elevated plus maze and context avoidance data obtained in the current studies was comparable to that described in our previous work [18], we first tested for significant differences between butyric acid or TMT-exposed rats from our previously published studies [18] against data obtained for the current studies using Student's $t$ tests. We found no differences in elevated plus maze open arm time for butyric acid-exposed rats $\left(t_{(50)}=0.0892, p=0.9293\right)$ or TMT-exposed rats $\left(t_{(88)}=0.6335, p=\right.$ 0.5088 ). Similarly, we found no difference in context avoidance for butyric acid-exposed rats $\left(t_{(50)}=0.2348, p=0.8145\right)$ or TMTexposed rats $\left(t_{(88)}=0.6380, p=0.5252\right)$. Since these behavioral data did not differ across studies, we segregated rats in the current studies according to our previously established algorithm [18], which defines all butyric acid-exposed rats as controls, and segregates predator odor-exposed rats into resilient and susceptible groups using cutoff criteria (time in open arms less than $50 \mathrm{~s}$ and $\Delta$ Paired less than $-20 \mathrm{~s}$; Fig. $1 \mathrm{~b}$ ).

To further validate these cutoff criteria within this study, we applied them to all rats and examined the distribution of rats meeting susceptible or resilient criteria. In line with our previous report [18], TMT-exposure was associated with significantly more rats meeting susceptible criteria relative to rats exposed to butyric acid (chi $=95.72, p<0.001$ ) (Fig. 1c). Although two butyric acidexposed rats met susceptible criteria, it is unlikely that these animals formed meaningful context aversion given that butyric acid is not fear-inducing [26]. Consistent with this, data from the two butyric acid-exposed rats that met susceptible criteria fell well within the range of data obtained for other control rats and not within the range observed for TMT-exposed, susceptible rats (data not shown). Based on this, we believe that the two butyric acidexposed rats that met susceptible criteria are not functionally susceptible, but rather met susceptible criteria due to a combination of individual differences in anxiety-like behavior $[27,28]$ and chance. This issue raises the possibility that TMTexposed rats could similarly meet susceptibility criteria due to individual differences that are independent of TMT-exposure. While possible, our previous work using the same segregation criteria indicated that susceptible rats displayed strong withingroup consistency in DA signaling and motivation for cocaine that significantly differed from both control and resilient rats [18].

\section{In vivo FSCV procedures}

FSCV experimentation began the day after stress and segregation testing. Rats were transferred to operant chambers (Med-Associate Inc.) housed within custom built sound-attenuated boxes where 
A

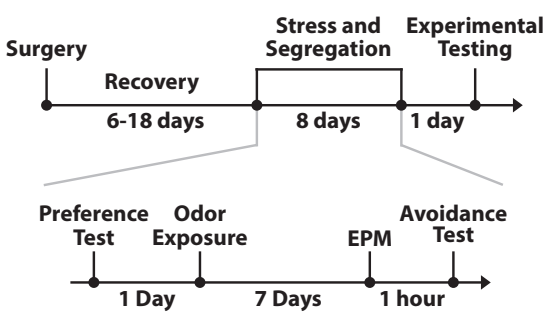

B

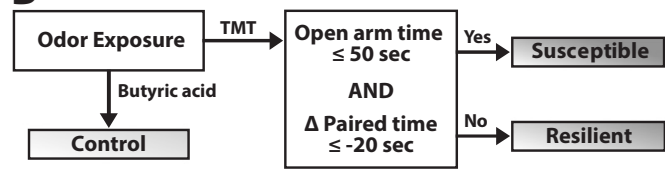

C

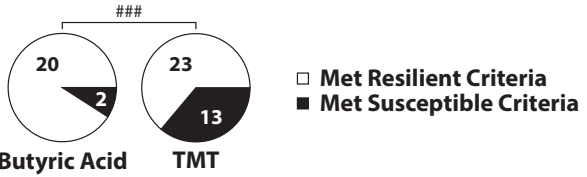

D

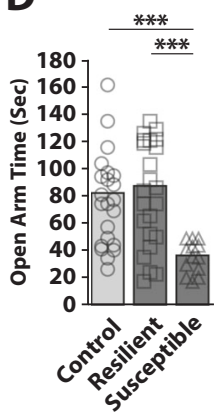

E

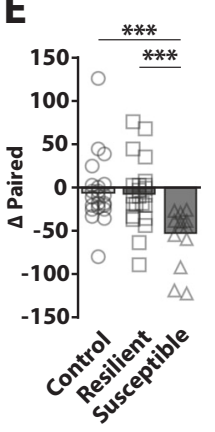

$\mathbf{F}$

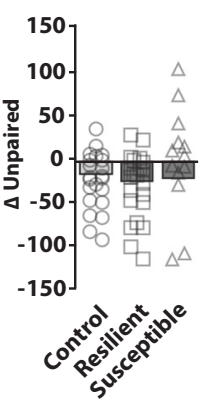

Fig. 1 Predator odor exposure stress produces two distinct populations. a Schematic of the stress and segregation procedure timeline. b Schematic of the segregation algorithm. c Distribution of butyric acid- and TMT-exposed rats that met susceptible criteria. Chi-square analysis showed a significant effect of TMT exposure on group distribution (chi $=95.72, p<0.001$ ). Note that butyric acid-exposed rats meeting susceptible criteria were nevertheless classified as controls. d Time spent in the open arms of the elevated plus maze (EPM). One-way ANOVA revealed a significant effect of group $\left(F_{(2,57)}=9.142, p<0.001\right)$. e Avoidance of the odor paired context chamber. One-way ANOVA revealed a significant effect of group $\left(F_{(2,57)}=9.121, p<0.001\right)$. $\mathbf{f}$ Change in time spent in the unpaired chamber. One-way ANOVA revealed no significant effects. Chi square: ${ }^{\# \# \#} p<0.001$. Bonferroni post hoc: ${ }^{* *} p<0.001$

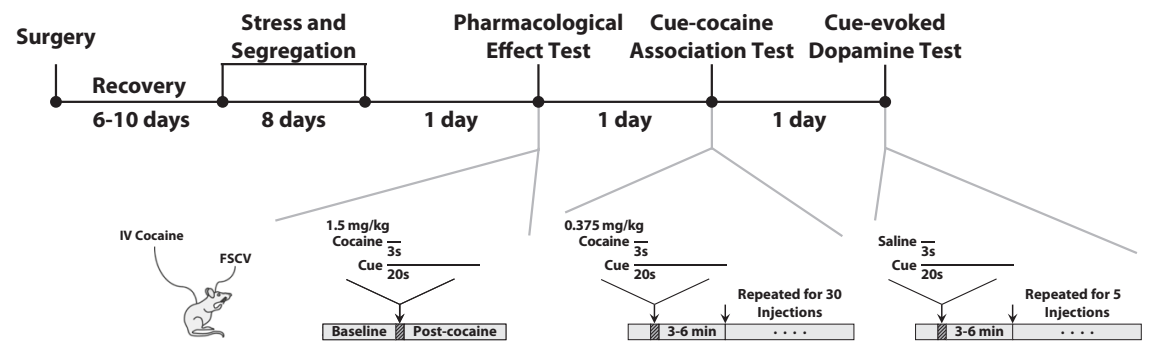

Fig. 2 Fast scan cyclic voltammetry experimental procedures. Schematic of the experimental design used for the FSCV experiments. Rats underwent surgery and then recovered for 6-10 days. Following recovery, rats underwent the stress and segregation protocol for control (butyric acid exposure) or stress (TMT exposure) conditions as previously described [18]. Three consecutive days of FSCV testing were carried out to examine the: (1) pharmacological effects of cocaine-Pharmacological Effect Test; (2) development of cue-evoked DA transients across cue-cocaine pairings-Cue-cocaine Association Test; and (3) the magnitude of persistent of cue-evoked DA transients associated with cocaine-Cue-evoked Dopamine Test

they were connected to voltammetry and infusion lines and allowed $1 \mathrm{~h}$ to acclimate. During this period, the carbon fiber electrode potential was linearly scanned $(-0.4$ to $1.3 \mathrm{~V}$ and back to $-0.4 \mathrm{~V}$ vs. $\mathrm{Ag} / \mathrm{AgCl}$ ) and cyclic voltammograms were assessed at the carbon fiber electrode every $100 \mathrm{~ms}$ with a scan rate of $400 \mathrm{~V} / \mathrm{s}$ using a voltammeter/amperometer (Electronics and Materials Engineering, Seattle, WA).

On the first day of FSCV testing, we examined DA transient frequency and magnitude at baseline and in response to cocaine in a "Pharmacological Effect Test" (Fig. 2). We recorded spontaneous DA transients for $6 \mathrm{~min}$ before administering a single IV injection of $1.5 \mathrm{mg} / \mathrm{kg}$ cocaine. Spontaneous DA transients were then recorded for an additional $5 \mathrm{~min}$ after cocaine delivery. On the second day of testing, we examined the development of cue-evoked DA transients across repeated cuecocaine pairings during a "Cue-cocaine Association Test" (Fig. 2). We used a single day procedure that has previously been shown to produce cue-evoked DA signals in the NAc [29]. Specifically, we paired IV infusions of $0.375 \mathrm{mg} / \mathrm{kg}$ cocaine with a 20 -s light and tone cue 30 times at a pseudo-random 3-6 min interval. On the third day of testing, we examined the persistence of cue-evoked DA signals following cue-cocaine association training on the previous day in a "Cue-evoked Dopamine Test" (Fig. 2). We exposed rats to the light and tone cue five times at a pseudorandom 3-6 min interval. Cue-evoked DA tests were conducted without administration of cocaine to test the persistence and magnitude of cue-elicited DA signals independent of the pharmacological effects of cocaine.

FSCV data analysis

Phasic DA transients were identified using Demon Voltammetry and Analysis software [30], and were based on previously described approaches [21,31]. Voltammograms from DA release events with amplitudes that exceeded five times the background 
noise were compared to a template cyclic voltammogram obtained from electrically evoked DA release in the NAc core of an awake rat. DA release events that displayed correlations greater than or equal to an $r^{2}=0.80$ relative to the template cyclic voltammogram were used for subsequent analysis.

DA transient magnitude was quantified by measuring area under the curve. Once a DA transient was identified, the outer bounds of each transient were defined as the first two points surrounding peak DA release with template cyclic voltammogram correlations less than $r^{2}=0.20$ (Supplemental Fig. 1). We then quantified DA concentration by dividing the area under the curve for each transient by an electrode calibration factor determined using an in situ calibration method as described previously [32] (Supplemental Fig. 2). This approach was used to measure DA transient magnitude for both spontaneous and cue-evoked DA signals.

For examination of spontaneous DA transients, we measured both frequency and magnitude of events. In addition, we built histograms of these data to more closely examine the relationship between groups [33]. For frequency, we averaged the number of inter-transient intervals into 1-s time bins across the duration of baseline recording $(6 \mathrm{~min})$. For magnitude, we binned DA transients into $0.1 \mathrm{nM}$ bins across the duration of baseline recording ( $6 \mathrm{~min})$ and then normalized these values by dividing each bin by the total number of DA transients observed $\left(N / N_{\text {Total }}\right)$ to account for any between group differences in DA transient frequency.

For examination of the rate of cue-evoked DA development, cue-evoked DA release data were analyzed by creating 30-s, perievent FSCV data files aligned to cue presentation such that $5 \mathrm{~s}$ preceded and $25 \mathrm{~s}$ followed the cue onset. These peri-event files were then averaged across 5 trials such that the 30 trials from the first day of testing were analyzed as 6 trial block averages (i.e., trials $1-5,6-10,11-15,16-20,21-25$, and 26-30) and the 5 trials from the second day of testing were analyzed as a single trial block average (i.e., trials $1-5$ ).

\section{Self-administration acquisition tests}

Acquisition of cocaine self-administration behavior was examined in a separate cohort of rats. Following stress and segregation testing, catheters were connected through a stainless-steel spring attached to a counterbalance and rats were provided access to cocaine on the following morning. Rats were given access to a lever within their home cage for $6 \mathrm{~h}$ (ZT 18-0) on each day of the 16-day acquisition period. Single lever presses resulted in the activation of a cue light placed directly above the lever and a single cocaine injection delivered over a $2-5$ s period. Cocaine injections were followed by a 20-s timeout period in which the lever was retracted. Extension of the lever after the timeout period served to cue the availability of cocaine. Injections of $0.09,0.19$, 0.38 , or $0.75 \mathrm{mg} / \mathrm{kg}$ doses were incrementally available for 4 consecutive daily sessions.

Self-administration behavior was analyzed only for rats that selfadministered at least five cocaine injections of each cocaine dose to ensure that all included rats adequately experienced each available dose. For example, a rat that lever pressed only one time on each of the 4 days of $0.09 \mathrm{mg} / \mathrm{kg}$ availability would not meet inclusion criteria (total lever presses =4) and would be excluded from all analyses. By comparison, a rat that lever pressed two times on each day would have met the inclusion criteria (total lever presses $=8$ ). In total, two control, two resilient, and one susceptible rat failed to meet this inclusion criteria and were thus removed from all analyses. Separate from the inclusion criteria, we defined the acquisition criteria to be used for post hoc analyses as 3 consecutive days of ten or more lever presses. Rats that lever pressed ten or more times on the final day of testing were allowed to continue self-administration for an additional 2 days to allow these rats to meet acquisition criteria. Rats that did not meet acquisition criteria were tested for catheter patency and were removed if patency was deemed insufficient, resulting in the removal of one control rat from analyses.

Statistics

Data were analyzed using IBM SPSS Statistics 24. Specific analyses are reported in respective figure captions.

\section{RESULTS}

Susceptible rats express context avoidance and increased anxietylike behavior

We first analyzed elevated plus maze and avoidance behavior of control, resilient, and susceptible rats using previously established cutoff criteria (Fig. 1b) [18]. These analyses revealed that, similar to our previous work [18], susceptible rats express increased anxiety like-behavior as measured by reduced open arm time in the elevated plus maze (Fig. 1d) and heightened avoidance of the odor-paired chamber (Fig. 1e), while resilient rats did not differ from controls across these measures. We also found that neither susceptible nor resilient rats differed from controls in nonspecific avoidance (Fig. 1f). When considered with our previous work [18], these results indicate that susceptible rats used in the current studies express a PTSD-like phenotype, while resilient rats do not.

Baseline phasic DA transient frequency is elevated in susceptible rats

We previously found that susceptible rats show increases in tonic DA levels at baseline [18]. However, the effects of trauma on baseline spontaneous, phasic DA signaling architecture remain unknown. To address this question, we first tested for differences in baseline spontaneous DA transient frequency across control, resilient, and susceptible rats. We found that average spontaneous DA transient frequency was elevated in susceptible rats but did not differ between control and resilient rats (Fig. 3a-c). In addition, we examined the magnitude of spontaneous DA release events in control, resilient, and susceptible rats. We found that DA transient magnitude did not differ across groups (Fig. 3a, d, e). These observations are consistent with our previous findings indicating that following traumatic stress exposure, susceptible rats exhibit increased extracellular DA at baseline [18], and suggest that a portion of this hyperdopaminergic phenotype is driven by increases in the rate at which spontaneous phasic DA transients occur.

The phasic DA response to cocaine is elevated in susceptible rats Cocaine is known to increase both the frequency and amplitude of spontaneous DA transients [34, 35], and cocaine-induced changes in spontaneous DA transient architecture are proposed to impact the development of cocaine use disorder [36, 37]. Importantly, while we and others have demonstrated that susceptible rats display enhanced sensitivity to the effects of cocaine using ex vivo FSCV and microdialysis $[18,38]$ it is unclear if this change in sensitivity influences phasic DA signaling in vivo. To address this, we carried out a "Pharmacological Effect Test" (see Fig. 2) to determine if susceptible rats express changes in spontaneous DA transient architecture following a single $1.5 \mathrm{mg} / \mathrm{kg}$ IV cocaine injection.

We found that the frequency of spontaneous DA transients was increased following cocaine in susceptible, resilient, and control rats (Fig. 3a, f), and that this increase in frequency did not differ across groups when expressed as a percent of baseline (Fig. $3 \mathrm{~g}$ ). Further, we found that cocaine increased average spontaneous DA transient magnitude in control, resilient, and susceptible rats (Fig. 3a, h), but that this increase was significantly higher in susceptible rats when expressed as a percent of baseline (Fig. 3i). Resilient rats did not differ from controls across any measure of cocaine effects on phasic DA transients. Together, these data indicate that while the processes that drive cocaine-induced increases in DA transient frequency are normal in susceptible rats, 


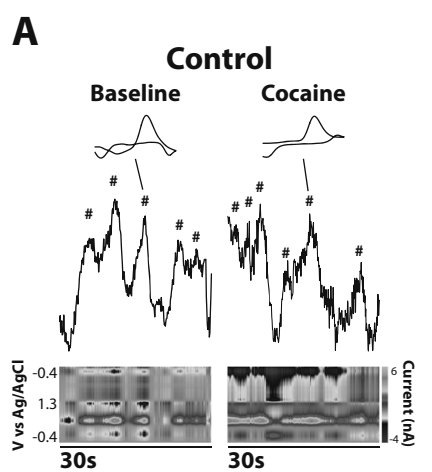

B
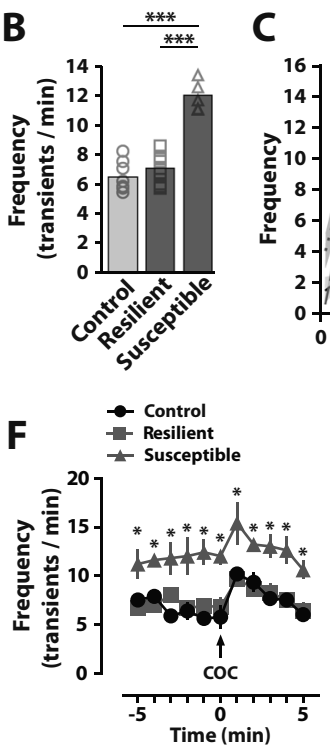

C

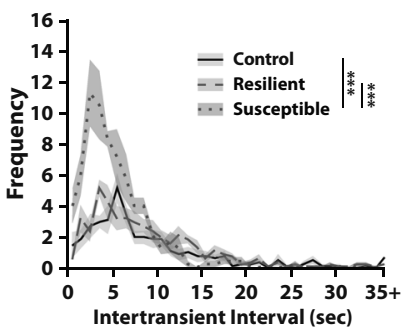

G

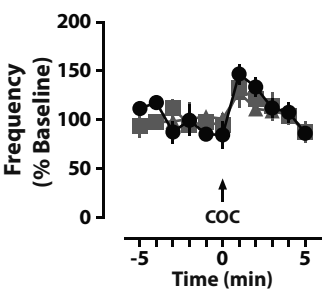

Resilient

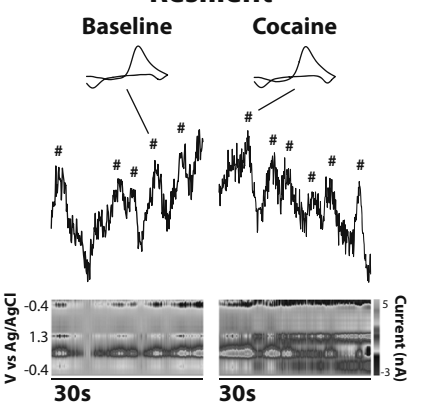

D

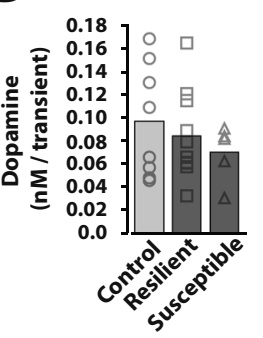

H

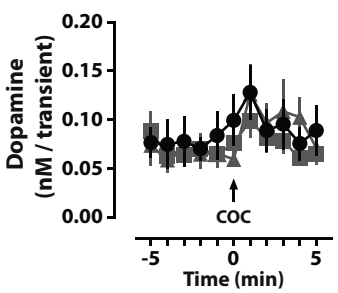

Susceptible

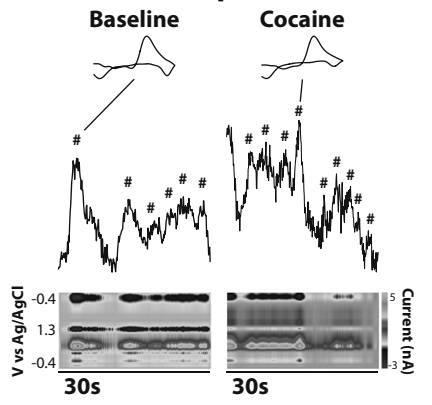

E

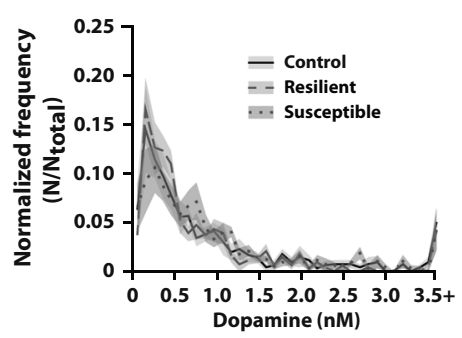

I

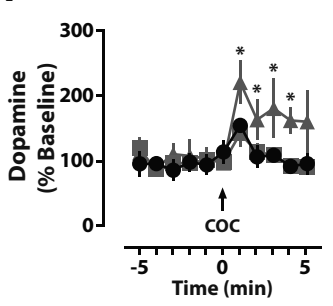

Fig. 3 Susceptible rats express changes in phasic DA signaling architecture before and after cocaine. a Color plots and current versus time plots for control, resilient, and susceptible rats at baseline and after cocaine delivery. DA transients are identified with pound signs, and example cyclic voltammograms for individual transients are shown above each current versus time plot. b Average DA transient frequency across the 6-min baseline recording session. One-way ANOVA revealed a significant effect of group $\left(F_{(2,22)}=52.74, p<0.001\right)$. c Intertransient interval histogram measured across the 6 -min baseline recording session. Between subjects two-way ANOVA with group as a betweensubjects measure and intertransient interval as the repeated measure revealed a significant effect of group $\left(F_{(2,715)}=25.91, p<0.001\right)$, interval $\left(F_{(35,715)}=41.03, p<0.001\right)$, and group $\times$ interval interaction $\left(F_{(70,715)}=5.910, p<0.001\right)$. Shaded regions indicate s.e.m. d Average DA transient magnitude measured across the 6-min baseline recording session. One-way ANOVA revealed no effect of group. e DA transient magnitude histogram normalized for frequency and measured across the 6-min baseline recording session. Between subjects two-way ANOVA with group as a between-subjects measure and DA magnitude as the repeated measure revealed a significant effect of magnitude $\left(F_{(35,720)}=27.14\right.$, $p<0.001)$, but no effect of group or interaction. Shaded regions indicate s.e.m. $\mathbf{f}$ Average DA transient frequency during baseline recording and after cocaine delivery. Two-way ANOVA with group as a between-subjects measure and time as the repeated measure revealed a significant effect of group $\left(F_{(2,205)}=106.7, p<0.001\right)$ and time $\left(F_{(10,205)}=5.461, p<0.001\right)$, but no interaction. $g$ Change in DA transient frequency calculated as a percent of baseline across baseline and after cocaine delivery. Two-way ANOVA with group as a between-subjects measure and time as the repeated measure revealed a significant effect of time $\left(F_{(10,170)}=5.095, p<0.001\right)$, but no effect of group or interaction. $\mathbf{h}$ Average DA transient frequency during baseline recording and after delivery of cocaine. Two-way ANOVA with group as a between-subjects measure and time as the repeated measure revealed a significant effect of time $\left(F_{(10,190)}=6.028, p<0.001\right)$, but no effect of group or interaction. $\mathbf{i}$ Change in DA transient magnitude calculated as a percent of baseline across baseline and after delivery of cocaine. Two-way ANOVA with group as a between-subjects measure and time as the repeated measure revealed a significant effect of group $\left(F_{(2,19)}=4.959, p<0.05\right)$, and time $\left(F_{(10,190)}=6.585, p<0.001\right)$, but no interaction. Control $n=8$, Resilient $n=10$, Susceptible $n=5$. Bonferroni post hoc: ${ }^{*} p<0.05 ;{ }^{* *} p<0.001$. COC $=1.5 \mathrm{mg} / \mathrm{kg}$ IV cocaine. Data are shown as mean ( \pm s.e.m.)

these rats nevertheless display enhanced cocaine effects on transient magnitude.

Cue-evoked DA signals develop more rapidly in susceptible rats Cue-evoked DA signals develop following repeated cue-cocaine pairings $[21,29]$, and similar cue-evoked DA transients have been shown to drive cocaine taking [16]. Furthermore, it has been proposed that cocaine-induced increases in spontaneous DA transient frequency and magnitude support the development of cue-evoked DA signals [36, 37]. To examine whether changes in DA transient architecture observed in susceptible rats correspond with changes in the rate of cue-evoked DA signal development we carried out a "Cue-cocaine Association Test" (see Fig. 2).

We used a noncontingent, cue-cocaine pairing procedure previously shown to produce cue-evoked DA signals in the NAc within a single session [29] to test cue-evoked DA signal development in control, resilient, and susceptible rats. We observed that cue-cocaine pairings elicited cue-evoked DA signals in all groups (Fig. 4a), but that susceptible rats developed cueevoked DA signals more rapidly than their resilient and control counterparts (Fig. 4a, b). In addition, we found that despite the more rapid development of these signals in susceptible rats, 
cue-evoked DA release did not differ across groups by the final trial block (trials 26-30) of the session (Fig. 4a, b). Next, we examined if the rate of change in DA signal magnitude varied throughout training across groups. Cue-evoked DA signal magnitudes, however, were not linear in all cases, with susceptible rats displaying decreases in DA signal magnitude in the fifth and sixth trial blocks (trials $21-25$ and $26-30$, respectively) relative to the fourth trial block (trials 16-20; Fig. 4c). This decrease may be attributable to differential activation of D2 auto receptors [39], cocaine-induced inhibition of DA release [40], or an as-of-yet undetermined mechanism. Thus, in order to avoid confounds associated with these factors, we calculated the rate of change in DA signal magnitude by performing a linear regression across the first through fourth trial blocks (trials 1-20; Fig. 4d). We found that susceptible rats displayed a greater rate of change in DA signal magnitude across these trial blocks (Fig. 4e), and that this rate of change in DA signal magnitude did not differ between control and resilient rats. Together, these observations indicate that cueevoked DA signals known to drive cocaine-taking behavior develop more rapidly in susceptible rats.

Cue-evoked DA signal persistence is increased in susceptible rats Exposure to cues previously paired with cocaine can initiate cocaine-taking behaviors [10, 11, 41], and it appears that cue-evoked DA signals are critical for the expression of cue-elicited cocaine seeking $[5,41]$. Thus, it is likely that persistent cue-evoked DA signals participate in continued cocaine seeking after initial usage. To examine the extent to which the magnitude of persistent cue-evoked DA signals differ across control, resilient, and susceptible rats on the day following cue-cocaine pairings, we performed a "Cue-evoked Dopamine Test" (see Fig. 2). We found that persistent cue-evoked DA signal magnitude was greater in susceptible rats than in their control and resilient counterparts and that DA signal magnitude did not differ between control and resilient rats (Fig. $4 f-h$ ). These findings indicate that cues previously paired with cocaine elicit stronger cue-evoked DA signals following initial pairings in susceptible rats.

Factors associated with increases in cue-evoked DA signal development and persistence

We next explored the relationship between baseline DA signaling and cocaine pharmacology with the rate of change and persistence of cue-evoked DA signals across rats in our FSCV studies. We used a stepwise multiple regression analysis to determine if the rate of change in cue-evoked DA signal magnitude was predicted by: (1) DA transient frequency at baseline; (2) DA transient magnitude at baseline; (3) peak DA transient frequency following cocaine; (4) peak DA transient magnitude following cocaine; (5) peak DA transient frequency following cocaine as a percent of baseline; or (6) peak DA transient
A

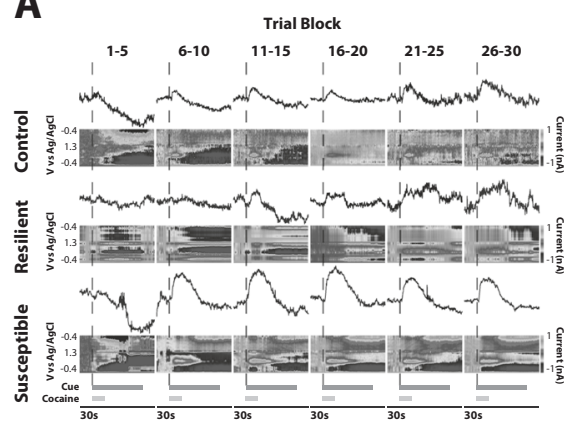

C

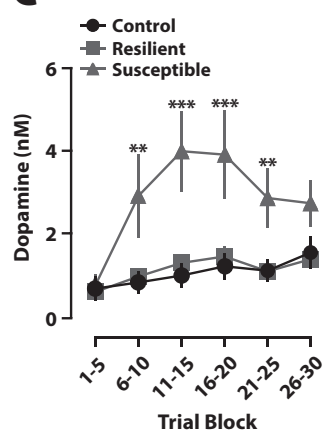

D

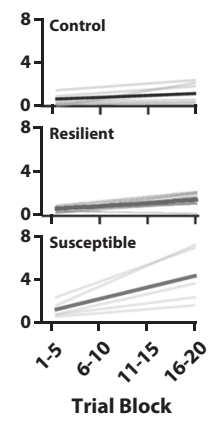

B

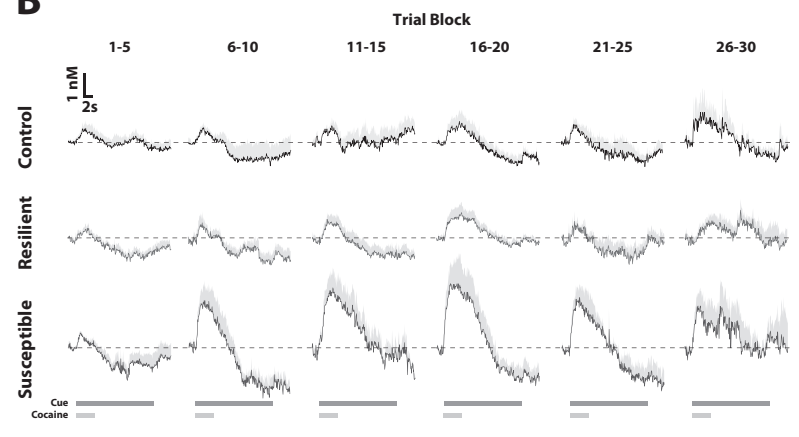

$\mathbf{E}$

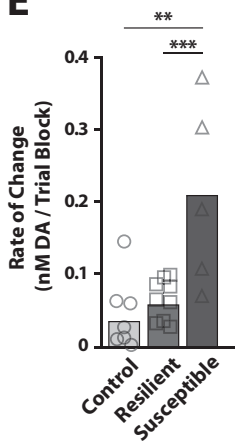

G<smiles>[Li]</smiles>

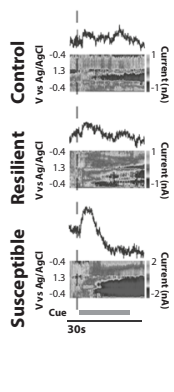

H

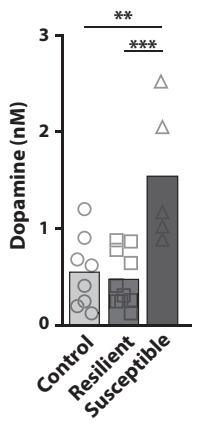

Fig. 4 Cue-evoked dopamine signals develop more rapidly and persist in susceptible rats. a Example color plots and current versus time plots for control, resilient, and susceptible rats for each trial block. Dashed vertical lines indicate cue and cocaine delivery onset. Horizontal green bars indicate the duration of the cue delivery. Horizontal blue bars indicate the duration of IV cocaine delivery. b Cue-evoked DA transient traces averaged for each trial block for control, resilient, and susceptible rats. c Average DA transient magnitude across trial blocks. Two-way ANOVA with group as the between-subjects measure and trial block as the repeated measure revealed a significant effect of group $\left(F_{(2,20)}=\right.$ $12.15, p<0.001)$, trial block $\left(F_{(5,100)}=10.25, p<0.001\right)$, and a group $\times$ trial block interaction $\left(F_{(10,100)}=3.137, p<0.01\right)$. d Regression lines used to calculate rate of change for control, resilient, and susceptible rats. Translucent lines depict individual rats and opaque lines represent the average regression line for each group. e Average rate of change in cue-evoked DA transients across the first through fourth trial blocks (trials $1-20)$ of the cue-cocaine association test day. One-way ANOVA revealed a significant effect of group $\left(F_{(2,20)}=12.13, p<0.001\right)$. $\mathbf{f}$ Example color plots and current versus time plots for control, resilient, and susceptible rats for the cue-evoked DA test. Dashed vertical lines indicate cue and cocaine delivery onset. Horizontal green bars indicate the duration of the cue delivery. g Persistent cue-evoked DA transient traces averaged across group for control, resilient, and susceptible rats. h Average magnitude of persistent cue-evoked DA signals. One-way ANOVA revealed a significant effect of group $\left(F_{(2,20)}=0.022, p<0.001\right)$. Control $n=8$, Resilient $n=10$, Susceptible $n=5$. Bonferroni post hoc: ${ }^{*} p<0.05$, ${ }^{* *} p<0.01$, ${ }_{* * *} p<0.001$. Data are shown as mean ( \pm s.e.m.) 
magnitude following cocaine as a percent of baseline (Supplemental Fig. 3). We found that only baseline DA transient frequency significantly predicted the rate of cue-evoked DA signal development (Supplemental Table 1). In addition, we examined if any features of phasic DA signaling at baseline or in response to cocaine predict the magnitude of persistent DA signals observed $24 \mathrm{~h}$ after cue-cocaine parings (Supplemental Fig. 4). We found that baseline DA transient frequency and peak cocaine-induced changes in DA transient magnitude both predict the magnitude of the persistent cue-evoked DA signal (Supplemental Table 2).

Phasic DA transients are related to DA neuron bursting [42], and thus measurements of baseline DA transient frequency may function as a readout of DA neuron burst activity [42, 43]. Considering this, our current results suggest that elevations in the processes that increase DA system burst activity in susceptible rats may also facilitate the development and persistence of cueevoked DA signals following cue-cocaine pairings. In addition, cocaine-induced increases in DA transient magnitude reflect the pharmacological effects of cocaine on DA release and uptake, and thus our regression analysis also suggests that elevations in the pharmacological effects of cocaine participate in the elevated magnitude of persistent cue-evoked DA signals observed in susceptible rats, but not in the rate at which these signals develop.

Susceptible rats display an elevated propensity to self-administer cocaine

In our FSCV experiments, we found that susceptible rats developed cue-evoked DA signals more rapidly than their control and resilient counterparts. Cue-evoked DA signals play an essential role in the initiation of cocaine-taking [16, 37], and thus it follows that increases in the rate and persistence of cue-evoked DA signals may confer an increase in the propensity to selfadminister cocaine. To examine this, we employed a modified extended access self-administration acquisition procedure [44, 45] that allows rats to escalate cocaine taking (Fig. 5a). We found that susceptible rats show increases in cocaine-reinforced lever pressing (Fig. 5b), and greater cumulative cocaine intake relative to control and resilient rats (Fig. 5c). In addition, we found that susceptible rats acquired cocaine self-administration faster than control and resilient rats (Fig. 5d).

To further examine these differences in acquisition, we referred to markers of addiction vulnerability in human populations. Specifically, human populations at risk for developing drug dependence show symptoms of addiction with fewer drug experiences than lower risk populations [46, 47]. We modeled this feature of addiction vulnerability by measuring the total amount of cocaine self-administered without reaching acquisition criteria across rats. We found that susceptible rats required less cocaine experience to acquire self-administration (Fig. 5e), but that resilient rats did not differ from controls. Lastly, humans who are vulnerable to developing cocaine use disorder transition from controlled drug taking to excessive drug use more rapidly than less vulnerable individuals [48]. To assess if susceptible rats transition to excessive cocaine taking more rapidly than their control and resilient counterparts, we compared cumulative cocaine intake after excluding all rats that did not meet acquisition criteria. We found that susceptible rats self-administer more cocaine over the 16-day testing period than the control and resilient rats (Fig. 5f). This latter observation suggests that susceptible rats more readily transition to excessive cocaine intake patterns relative to control and resilient rats.

\section{DISCUSSION}

The current studies demonstrate that susceptibility to traumatic stress is associated with differences in spontaneous phasic DA signaling architecture both before and after the administration of cocaine. Specifically, we found that the high spontaneous DA frequency rate and high sensitivity to the DA-elevating effects of cocaine observed in susceptible rats are associated with the rapid development of high magnitude cue-evoked DA signals. Further, we demonstrated that susceptible rats exhibit a heightened propensity to self-administer cocaine. Together, these observations demonstrate the co-occurrence of trauma susceptibility with cocaine use vulnerability and suggest that differences in phasic DA signaling architecture may contribute to the process by which this vulnerability occurs.

Importantly, when viewed within the framework of our previous report [18], our current observations enhance our understanding of the DA dynamics underlying cocaine use vulnerability in susceptible rats. For example, given that phasic DA transients have been shown to significantly contribute to DA tone [38], the current finding that susceptible rats display increased DA transient activity is consistent with our previous observation that susceptible rats exhibit increased extracellular DA in the NAc [18].

Interestingly, the lack of difference in spontaneous DA transient magnitude between susceptible, resilient, and control rats observed in the current studies (Fig. 3), may at first appear to contradict our previous observations [18]. Specifically, in our previous ex vivo studies we observed low electrically-stimulated DA release in susceptible rats, suggesting the possibility that spontaneous DA transient magnitude in vivo would be similarly reduced. Nevertheless, in our previous ex vivo studies we also observed reduced DA uptake rate in susceptible rats which suggests an increase in spontaneous DA transient magnitude [18]. Thus, the co-occurrence of these opposing processes in susceptible rats would result in no changes to spontaneous DA transient magnitude as observed in the current studies in freely moving animals.

Spontaneous phasic DA signals are believed to be a product of DA neuron bursting $[42,43]$. Thus, the most likely explanation for susceptible rats displaying increases in spontaneous phasic DA signal frequency is that susceptibility to traumatic stress coincides with increases in DA neuron burst activity. DA neuron bursting is a product of glutamatergic inward calcium currents [49-52], and these same currents play a significant role in synaptic plasticity events that are required for the development of cue-evoked DA signals and Pavlovian learning [53, 54]. Consistent with these observations, we found that phasic DA transient frequency correlates with the rate of development and the magnitude of persistent cue-evoked DA signals. Together, this evidence suggests that a common mechanism may enhance excitatory signaling onto DA neurons to drive increases in baseline DA signaling and cue-evoked DA signaling in susceptible rats. Continued efforts are focused on elucidating such a mechanism.

We also found that susceptible rats displayed exaggerated cocaine-induced increases in phasic DA transient magnitude. It has been hypothesized that cocaine-induced elevations in extrasynaptic DA may influence the rate at which phasic DA signals entrain to drug-associated cues. Specifically, it has been shown that repeated drug-induced increases in extra-synaptic DA trigger a series of molecular and physiological adaptations across learning and memory circuits [55-57]. These physiological changes include enhanced synaptic input onto DA neurons of the VTA [58-60], and such increases in synaptic strength are a critical factor in reward learning $[53,57]$. In our previous work, we found that susceptible rats express heightened DA terminal cocaine sensitivity, such that cocaine is more effective at blocking DA uptake at the level of DA terminals [18]. Consistent with these prior studies, here we found that the magnitude of persistent cue-evoked DA signals is higher in susceptible rats. Furthermore, we found that the effect of cocaine on DA transient magnitude predicts increases in the magnitude of persistent cue-evoked DA signals. Together, this evidence suggests that increases in DA terminal cocaine sensitivity may drive enhanced cocaine effects on extra-synaptic DA in vivo, and that this change in cocaine pharmacology participates in 
A

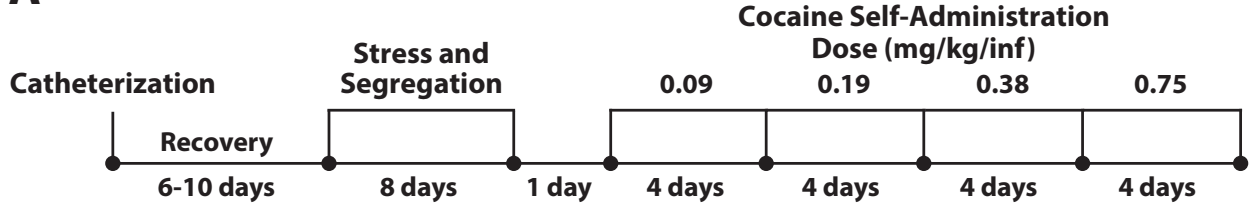

B

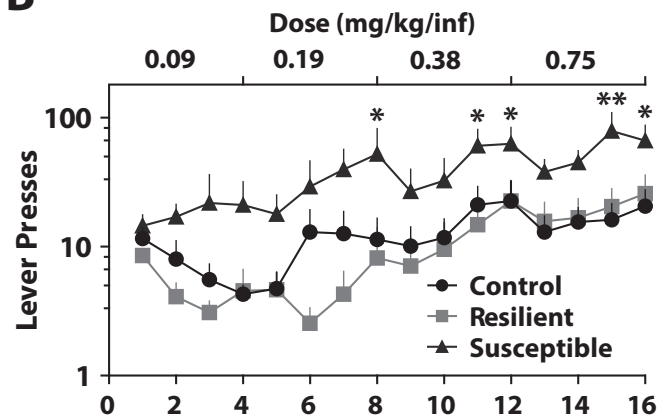

C

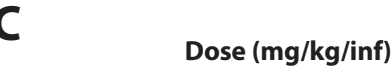

$\begin{array}{llll}0.09 & 0.19 & 0.38 & 0.75\end{array}$
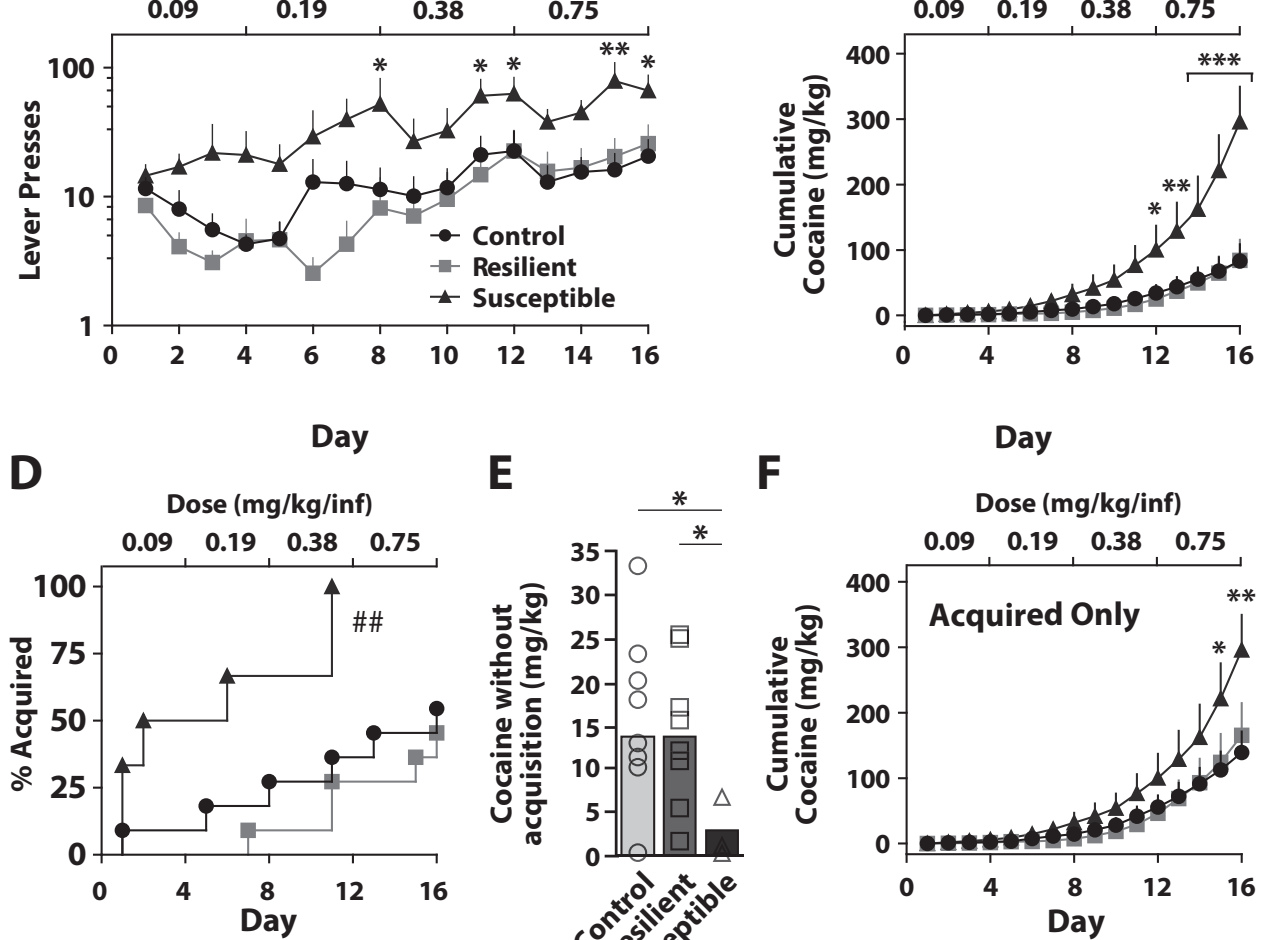

$\mathbf{E}$

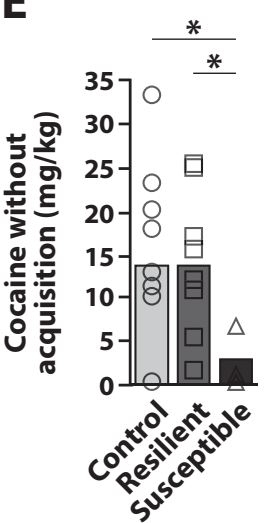

$\mathbf{F}$

Day

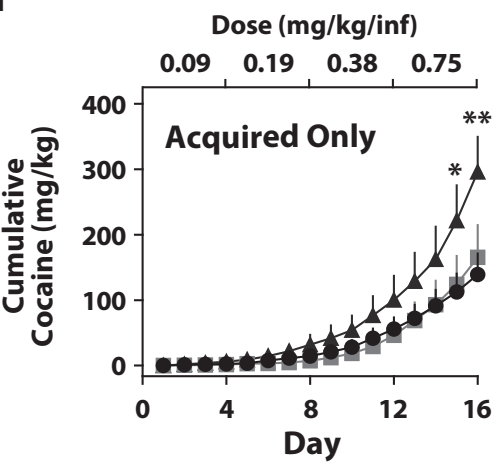

Fig. 5 Susceptible rats display an increased propensity to self-administer cocaine. a Experimental timeline for cocaine self-administration acquisition experiments. b Number of lever presses made across the 16-day test period. Two-way ANOVA with group as the between-subjects measure and day as the repeated measure revealed a significant effect of group $\left(F_{(2.375)}=7.671, p<0.01\right)$, day $\left(F_{(15.375)}=7.671, p<0.001\right)$, and a group $\times$ day interaction $\left(F_{(30,375)}=1.555, p<0.001\right)$. c Cumulative cocaine self-administered across the 16-day test period. Two-way ANOVA with group as the between-subjects measure and day as the repeated measure revealed a significant effect of group $\left(F_{(2,375)}=5.92, p<0.01\right)$, day $\left(F_{(15,375)}=38.33, p<0.001\right)$, and group $\times$ day interaction $\left(F_{(30,375)}=5.696, p<0.01\right)$. d Percent of animals that met acquisition criteria across testing days. Log-rank Mantel-Cox (chi square $=11.79, p=0.003$ ). e Amount of cocaine self-administered without reaching acquisition criteria. One-way ANOVA revealed a significant effect of group $\left(F_{(2,25)}=4.63, p<0.05\right)$. $\mathbf{f}$ Cumulative cocaine self-administered by rats that met acquisition criteria. Two-way ANOVA with group as the between-subjects measure and day as the repeated measure revealed a significant effect of day $\left(F_{(15,210)}=33.69, p<0.001\right)$, and group $\times$ day interaction $\left(F_{(30,210)}=1.57, p<0.05\right)$. b-e Control $n=11$, Resilient $n=11$, Susceptible $n=6$. f Control $n=6$, Resilient $n=5$, Susceptible $n=6$. Bonferroni post hoc: ${ }^{*} p<0.05,{ }^{* *} p<0.01{ }^{* * *} p<0.001$ susceptible versus control and resilient. Log rank (Mantel-Cox) ${ }^{\# \#} p<0.01$. Data are shown as mean ( \pm s.e.m.)

determining the magnitude of cocaine-associated phasic DA signals in susceptible populations.

Cue-evoked DA signals are known to influence cue-initiated cocaine taking $[10,11,16]$. Indeed, the magnitude of cue-evoked DA signals positively correlate with the vigor of cue-elicited cocaine seeking [11], and disrupting DA signaling in the NAc suppresses cocaine seeking [61]. Given that we found elevations in the rapidity of development and the magnitude of persistent cue-evoked DA signals in susceptible rats, we predicted that these rats would rapidly develop cocaine self-administration behaviors. We found that susceptible rats displayed increases in the rate of cocaine selfadministration acquisition, and that susceptible rats transitioned to excessive cocaine taking more readily than control and resilient rats. Using a similar model, others have demonstrated that susceptible rats show increases in cue-induced reinstatement of cocaine seeking [20], and together with our current data this suggests that susceptible rats may express a generally heightened sensitivity to cocaine-paired cues. It is important to note, however, that the behavioral contribution of heightened cue sensitivity versus more general changes in DA system activity and cocaine pharmacology remain unclear. Ongoing studies seek to parse the distinct influence that each of these features imparts on cocaine use vulnerability following trauma.

The finding that susceptible rats rapidly transition to excessive cocaine taking is particularly interesting and may appear to contradict our previous finding that susceptible rats exhibit a reduction in cocaine consumption at null cost [18]. It should be noted, however, that rats in our earlier studies were trained under conditions that do not promote escalation of cocaine taking $[18,62]$, whereas rats in the current work self-administered 
cocaine under conditions that do promote escalation [45, 63]. With this consideration, it is likely that susceptible rats express a reduction in cocaine consumption on initial usage but are nevertheless more likely to escalate cocaine taking. Indeed, others have found that that low initial levels of cocaine consumption predict a heightened propensity to escalate cocaine taking [64]. Together, these observations support the hypothesis that susceptible rats express a drug-use vulnerable phenotype in which enhanced cocaine sensitivity on initial usage predicts early acquisition of self-administration and the rapid transition to excessive cocaine taking.

The degree to which the dopaminergic and cocaine use vulnerability profile observed in susceptible subjects is directly related to trauma exposure remains unknown. While stress has been demonstrated to directly increase DA neuron excitability, mesolimbic DA system output, and cocaine sensitivity [65-71], other evidence indicates that susceptibility can be attributed to inherent, preexisting features that coincide with susceptibility. Indeed, pre-existing genetic [72], hormonal [73-75], pharmacological [76, 77], and physiological [78] indices can predict susceptibility versus resilience to stress. Thus, while some forms of stress may directly alter DA signaling, it is nevertheless possible that, in our model, pre-existing mesolimbic DA system hyperactivity coincides with or contributes to stress susceptibility.

Overall, we provide evidence that susceptible rats express heightened DA system activity with an enhanced DA response to the pharmacological effects of cocaine, and that these adaptations in DA system function may participate in the rapid development of persistent cue-evoked DA signals. As expected, this dopaminergic phenotype corresponded with increases in the development of excessive cocaine-taking behaviors. Together, our findings suggest that trauma-associated differences in DA system function participate in the cocaine use vulnerability observed in PTSD populations and contribute to our understanding of the dopaminergic factors underlying cocaine use vulnerability.

\section{FUNDING AND DISCLOSURE}

The authors report no biomedical financial interests or potential conflicts of interest. This work was supported by grant DA031900 to R.A.E., and F31-DA042505 Z.D.B.

\section{ADDITIONAL INFORMATION}

Supplementary Information accompanies this paper at (https://doi.org/10.1038/ s41386-019-0526-1).

Publisher's note Springer Nature remains neutral with regard to jurisdictional claims in published maps and institutional affiliations.

\section{REFERENCES}

1. Kessler RC, Sonnega A, Bromet E, Hughes M, Nelson CB. Posttraumatic stress disorder in the National Comorbidity Survey. Arch Gen Psychiatry. 1995; 52:1048-60.

2. Back S, Dansky BS, Coffey SF, Saladin ME, Sonne S, Brady KT. Cocaine dependence with and without post-traumatic stress disorder: a comparison of substance use, trauma history and psychiatric comorbidity. Am J Addict. 2000;9:51-62.

3. Khoury L, Tang YL, Bradley B, Cubells JF, Ressler KJ. Substance use, childhood traumatic experience, and Posttraumatic Stress Disorder in an urban civilian population. Depress anxiety. 2010;27:1077-86

4. Chilcoat HD, Breslau N. Posttraumatic stress disorder and drug disorders: testing causal pathways. Arch Gen Psychiatry. 1998;55:913-7.

5. Volkow ND, Wang GJ, Telang F, Fowler JS, Logan J, Childress AR, et al. Cocaine cues and dopamine in dorsal striatum: mechanism of craving in cocaine addiction. J Neurosci. 2006;26:6583-8.

6. Everitt BJ, Parkinson JA, Olmstead MC, Arroyo M, Robledo P, Robbins TW. Associative processes in addiction and reward. The role of amygdala-ventral striatal subsystems. Ann N Y Acad Sci. 1999;877:412-38.
7. Volkow ND, Morales M. The brain on drugs: from reward to addiction. Cell. 2015;162:712-25.

8. Saladin ME, Drobes DJ, Coffey SF, Dansky BS, Brady KT, Kilpatrick DG. PTSD symptom severity as a predictor of cue-elicited drug craving in victims of violent crime. Addict Behav. 2003;28:1611-29.

9. Regier PS, Monge ZA, Franklin TR, Wetherill RR, Teitelman A, Jagannathan $\mathrm{K}$, et al. Emotional, physical and sexual abuse are associated with a heightened limbic response to cocaine cues. Addict Biol. 2017;22:1768-77.

10. LeBlanc $\mathrm{KH}$, Ostlund SB, Maidment NT. Pavlovian-to-instrumental transfer in cocaine seeking rats. Behav Neurosci. 2012;126:681-9.

11. Ostlund SB, LeBlanc KH, Kosheleff AR, Wassum KM, Maidment NT. Phasic mesolimbic dopamine signaling encodes the facilitation of incentive motivation produced by repeated cocaine exposure. Neuropsychopharmacology. 2014;39:2441-9.

12. Everitt BJ, Robbins TW. From the ventral to the dorsal striatum: devolving views of their roles in drug addiction. Neurosci Biobehav Rev. 2013;37:1946-54.

13. Ito R, Robbins TW, Everitt BJ. Differential control over cocaine-seeking behavior by nucleus accumbens core and shell. Nat Neurosci. 2004;7:389-97.

14. Fuchs RA, Evans KA, Parker MC, See RE. Differential involvement of the core and shell subregions of the nucleus accumbens in conditioned cue-induced reinstatement of cocaine seeking in rats. Psychopharmacology. 2004;176:459-65.

15. Di Ciano P, Robbins TW, Everitt BJ. Differential effects of nucleus accumbens core, shell, or dorsal striatal inactivations on the persistence, reacquisition, or reinstatement of responding for a drug-paired conditioned reinforcer. Neuropsychopharmacology. 2008;33:1413-25.

16. Phillips PE, Stuber GD, Heien ML, Wightman RM, Carelli RM. Subsecond dopamine release promotes cocaine seeking. Nature. 2003;422:614-8.

17. Stuber GD, Wightman RM, Carelli RM. Extinction of cocaine self-administration reveals functionally and temporally distinct dopaminergic signals in the nucleus accumbens. Neuron. 2005;46:661-9.

18. Brodnik ZD, Black EM, Clark MJ, Kornsey KN, Snyder NW, España RA. Susceptibility to traumatic stress sensitizes the dopaminergic response to cocaine and increases motivation for cocaine. Neuropharmacology. 2017;125:295-307.

19. Arena DT, Covington HE III, DeBold JF, Miczek KA. Persistent increase of I.V. cocaine self-administration in a subgroup of $\mathrm{C57BL} / 6 \mathrm{~J}$ male mice after social defeat stress. Psychopharmacology. 2019;236:2027-37.

20. Schwendt M, Shallcross J, Hadad NA, Namba MD, Hiller H, Wu L, et al. A novel rat model of comorbid PTSD and addiction reveals intersections between stress susceptibility and enhanced cocaine seeking with a role for mGlu5 receptors. Transl Psychiatry. 2018;8:209.

21. Levy KA, Brodnik ZD, Shaw JK, Perrey DA, Zhang Y, España RA. Hypocretin receptor 1 blockade produces bimodal modulation of cocaine-associated mesolimbic dopamine signaling. Psychopharmacology. 2017;234:2761-76.

22. Clark JJ, Sandberg SG, Wanat MJ, Gan JO, Horne EA, Hart AS, et al. Chronic microsensors for longitudinal, subsecond dopamine detection in behaving animals. Nat Methods. 2010;7:126-9.

23. Cohen H, Kozlovsky N, Alona C, Matar MA, Joseph Z. Animal model for PTSD: from clinical concept to translational research. Neuropharmacology. 2012;62:715-24.

24. Cohen H, Zohar J, Matar MA, Zeev K, Loewenthal U, Richter-Levin G. Setting apart the affected: the use of behavioral criteria in animal models of post traumatic stress disorder. Neuropsychopharmacology. 2004;29:1962-70.

25. File SE, Lippa AS, Beer B, Lippa MT. Animal tests of anxiety. Curr Protoc Neurosci. 2004. Chapter 8: p. Unit 8.3.

26. Fendt M, Endres T. 2,3,5-Trimethyl-3-thiazoline (TMT), a component of fox odorjust repugnant or really fear-inducing? Neurosci Biobehav Rev. 2008;32:1259-66.

27. Feyissa DD, Aher YD, Engidawork E, Hoger H, Lubec G, Korz V. Individual differences in male rats in a behavioral test battery: a multivariate statistical approach. Front Behav Neurosci. 2017;11:26.

28. Curé M, Rolinat JP. Behavioral heterogeneity in Sprague-Dawley rats. Physiol Behav. 1992;51:771-4.

29. Aragona BJ, Day JJ, Roitman MF, Cleaveland NA, Wightman RM, Carelli RM. Regional specificity in the real-time development of phasic dopamine transmission patterns during acquisition of a cue-cocaine association in rats. Eur J Neurosci. 2009;30:1889-99.

30. Yorgason JT, España RA, Jones SR. Demon voltammetry and analysis software: analysis of cocaine-induced alterations in dopamine signaling using multiple kinetic measures. J Neurosci Methods. 2011;202:158-64.

31. Robinson DL, Wightman RM. Nomifensine amplifies subsecond dopamine signals in the ventral striatum of freely-moving rats. J Neurochem. 2004;90:894-903.

32. Roberts JG, Toups JV, Eyualem E, McCarty GS, Sombers LA. In situ electrode calibration strategy for voltammetric measurements in vivo. Anal Chem. 2013;85:11568-75.

33. Howard CD, Daberkow DP, Ramsson ES, Keefe KA, Garris PA. Methamphetamineinduced neurotoxicity disrupts naturally occurring phasic dopamine signaling. Eur J Neurosci. 2013;38:2078-88. 
34. Aragona BJ, Cleaveland NA, Stuber GD, Day JJ, Carelli RM, Wightman RM. Preferential enhancement of dopamine transmission within the nucleus accumbens shell by cocaine is attributable to a direct increase in phasic dopamine release events. J Neurosci. 2008;28:8821-31.

35. Stuber GD, Roitman MF, Phillips PEM, Carelli RM, Wightman RM. Rapid dopamine signaling in the nucleus accumbens during contingent and noncontingent cocaine administration. Neuropsychopharmacology. 2004;30:853-63.

36. Covey DP, Roitman MF, Garris PA. Illicit dopamine transients: reconciling actions of abused drugs. Trends Neurosci. 2014;37:200-10.

37. Willuhn I, Burgeno LM, Groblewski PA, Phillips PE. Excessive cocaine use results from decreased phasic dopamine signaling in the striatum. Nat Neurosci. 2014;17:704-9.

38. Shimamoto A, Holly EN, Boyson CO, DeBold JF, Miczek KA. Individual differences in anhedonic and accumbal dopamine responses to chronic social stress and their link to cocaine self-administration in female rats. Psychopharmacology. 2015;232:825-34.

39. Dreyer JK, Hounsgaard J. Mathematical model of dopamine autoreceptors and uptake inhibitors and their influence on tonic and phasic dopamine signaling. J Neurophysiol. 2013;109:171-82.

40. Federici M, Latagliata EC, Ledonne A, Rizzo FR, Feligioni M, Sulzer D, et al. Paradoxical abatement of striatal dopaminergic transmission by cocaine and methylphenidate. J Biol Chem. 2014;289:264-74.

41. Saunders BT, Yager LM, Robinson TE. Cue-evoked cocaine "craving": role of dopamine in the accumbens core. J Neurosci. 2013;33:13989-4000.

42. Sombers LA, Beyene M, Carelli RM, Wightman RM. Synaptic overflow of dopamine in the nucleus accumbens arises from neuronal activity in the ventral tegmental area. J Neurosci. 2009;29:1735-42.

43. Owesson-White CA, Roitman MF, Sombers LA, Belle AM, Keithley RB, Peele JL, et al. Sources contributing to the average extracellular concentration of dopamine in the nucleus accumbens. J Neurochem. 2012;121:252-62.

44. Goeders NE. The HPA axis and cocaine reinforcement. Psychoneuroendocrinology. 2002;27:13-33.

45. Mantsch J, Ho A, Schlussman S, Kreek M. Predictable individual differences in the initiation of cocaine self-administration by rats under extended-access conditions are dose-dependent. Psychopharmacology. 2001;157:31-9.

46. Vsevolozhskaya OA, Anthony JC. Transitioning from first drug use to dependence onset: Illustration of a multiparametric approach for comparative epidemiology. Neuropsychopharmacology. 2016;41:869-76.

47. Vsevolozhskaya OA, Anthony JC. Estimated probability of becoming a case of drug dependence in relation to duration of drug-taking experience: a functional analysis approach. Int J Methods Psychiatr Res. 2016;26:e1513.

48. Siegel RK. Changing patterns of cocaine use: longitudinal observations, consequences, and treatment. NIDA Res Monogr. 1984;50:92-110.

49. Canavier CC, Landry RS. An increase in AMPA and a decrease in SK conductance increase burst firing by different mechanisms in a model of a dopamine neuron in vivo. J Neurophysiol. 2006;96:2549-63.

50. Chergui K, Charlety PJ, Akaoka H, Saunier CF, Brunet JL, Buda M, et al. Tonic activation of NMDA receptors causes spontaneous burst discharge of rat midbrain dopamine neurons in vivo. Eur J Neurosci. 1993;5:137-44.

51. Johnson SW, Seutin V, North RA. Burst firing in dopamine neurons induced by Nmethyl-D-aspartate: role of electrogenic sodium pump. Science. 1992;258:665-7.

52. Kuznetsov AS, Kopell NJ, Wilson CJ. Transient high-frequency firing in a coupledoscillator model of the mesencephalic dopaminergic neuron. J Neurophysiol. 2006;95:932-47.

53. Stuber GD, Klanker M, de Ridder B, Bowers MS, Joosten RN, Feenstra MG, et al. Reward-predictive cues enhance excitatory synaptic strength onto midbrain dopamine neurons. Science. 2008;321:1690-2.

54. Zweifel LS, Fadok JP, Argilli E, Garelick MG, Jones GL, Dickerson TM, et al. Activation of dopamine neurons is critical for aversive conditioning and prevention of generalized anxiety. Nat Neurosci. 2011;14:620-6.

55. Berke JD, Hyman SE. Addiction, dopamine, and the molecular mechanisms of memory. Neuron. 2000;25:515-32.
56. Dong $Y$, Nestler EJ. The neural rejuvenation hypothesis of cocaine addiction Trends Pharm Sci. 2014;35:374-83.

57. Stuber GD, Hopf FW, Tye KM, Chen BT, Bonci A. Neuroplastic alterations in the limbic system following cocaine or alcohol exposure. Curr Top Behav Neurosci. 2010;3:3-27.

58. Chen BT, Bowers MS, Martin M, Hopf FW, Guillory AM, Carelli RM, et al. Cocaine but not natural reward self-administration nor passive cocaine infusion produces persistent LTP in the VTA. Neuron. 2008;59:288-97.

59. Ungless MA, Whistler JL, Malenka RC, Bonci A. Single cocaine exposure in vivo induces long-term potentiation in dopamine neurons. Nature. 2001;411:583-7.

60. Wanat MJ, Bonci A. Dose-dependent changes in the synaptic strength on dopamine neurons and locomotor activity after cocaine exposure. Synapse. 2008;62:790-5.

61. Roberts DC, Koob GF, Klonoff P, Fibiger HC. Extinction and recovery of cocaine self-administration following 6-hydroxydopamine lesions of the nucleus accumbens. Pharmacol Biochem Behav. 1980;12:781-7.

62. Roberts AJ, Koob GF. The neurobiology of addiction: an overview. Alcohol Health Res World. 1997;21:101-6.

63. Ahmed SH, Koob GF. Transition from moderate to excessive drug intake: change in hedonic set point. Science. 1998;282:298-300.

64. Bentzley BS, Jhou TC, Aston-Jones G. Economic demand predicts addiction-like behavior and therapeutic efficacy of oxytocin in the rat. Proc Natl Acad Sci USA 2014;111:11822-7.

65. Miczek KA, Nikulina EM, Shimamoto A, Covington HE III. Escalated or suppressed cocaine reward, tegmental BDNF, and accumbal dopamine caused by episodic versus continuous social stress in rats. J Neurosci. 2011:31:9848-57.

66. Tidey JW, Miczek KA. Acquisition of cocaine self-administration after social stress: role of accumbens dopamine. Psychopharmacology. 1997;130:203-12.

67. Garcia-Keller C, Martinez SA, Esparza MA, Bollati F, Kalivas PW, Cancela LM Cross-sensitization between cocaine and acute restraint stress is associated with sensitized dopamine but not glutamate release in the nucleus accumbens. Eur J Neurosci. 2013;37:982-95.

68. Sorg BA, Kalivas PW. Effects of cocaine and footshock stress on extracellular dopamine levels in the medial prefrontal cortex. Neuroscience. 1993;53: 695-703.

69. Saal D, Dong Y, Bonci A, Malenka RC. Drugs of abuse and stress trigger a common synaptic adaptation in dopamine neurons. Neuron. 2003;37:577-82.

70. Cao JL, Covington HE III, Friedman AK, Wilkinson MB, Walsh JJ, Cooper DC, et al. Mesolimbic dopamine neurons in the brain reward circuit mediate susceptibility to social defeat and antidepressant action. J Neurosci. 2010;30:16453-8.

71. Friedman A. Neuroscience. Jump-starting natural resilience reverses stress susceptibility. Science. 2014;346:555.

72. Chandra R, Francis TC, Nam H, Riggs LM, Engeln M, Rudzinskas S, et al. Reduced Slc6a15 in nucleus accumbens D2-neurons underlies stress susceptibility. J Neurosci. 2017;37:6527-38.

73. Whitaker AM, Farooq MA, Edwards S, Gilpin NW. Post-traumatic stress avoidance is attenuated by corticosterone and associated with brain levels of steroid receptor co-activator-1 in rats. Stress. 2016;19:69-77.

74. Whitaker AM, Gilpin NW. Blunted hypothalamo-pituitary adrenal axis response to predator odor predicts high stress reactivity. Physiol Behav. 2015;147:16-22.

75. Danan D, Matar MA, Kaplan Z, Zohar J, Cohen H. Blunted basal corticosterone pulsatility predicts post-exposure susceptibility to PTSD phenotype in rats. Psychoneuroendocrinology. 2018;87:35-42.

76. Nam H, Chandra R, Francis TC, Dias C, Cheer JF, Lobo MK. Reduced nucleus accumbens enkephalins underlie vulnerability to social defeat stress. Neuropsychopharmacology. 2019;44:1876-85.

77. Wook Koo J, Labonte B, Engmann O, Calipari ES, Juarez B, Lorsch Z, et al. Essential role of mesolimbic brain-derived neurotrophic factor in chronic social stressinduced depressive behaviors. Biol Psychiatry. 2016;80:469-78.

78. Muir J, Lorsch ZS, Ramakrishnan C, Deisseroth K, Nestler EJ, Calipari ES, et al. In vivo fiber photometry reveals signature of future stress susceptibility in nucleus accumbens. Neuropsychopharmacology. 2018;43:255-63. 